\title{
A mutational history of Alzheimer's
}

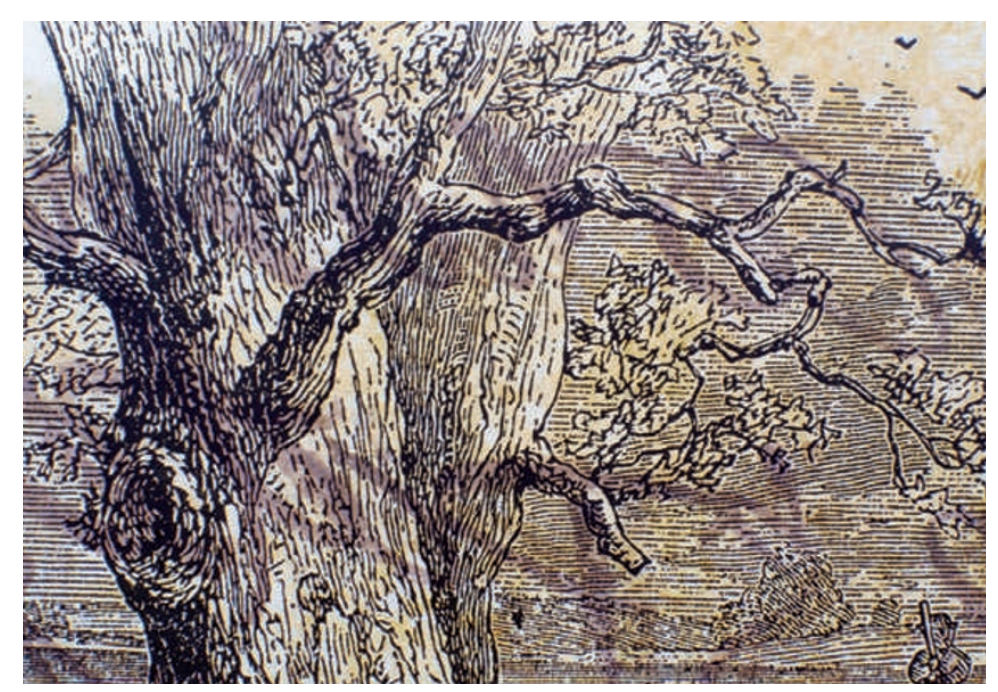

A common polymorphism that accelerates the onset of the most common form of Alzheimer's disease (AD) by an average of 7 years has been identified. The polymorphism in question - a poly( $\mathrm{T})$ variant in a mitochondrial translocase gene - was identified using a new phylogenetic mapping approach and only affects individuals who carry the $\varepsilon 3$ allele of the $\mathrm{AD}$-susceptibility gene, APOE.

Late-onset AD (LOAD) accounts for over $95 \%$ of $\mathrm{AD}$ cases in people over 60-65 years of age. Previous studies had implicated a region on chromosome 19 containing three genes in susceptibility to the disease, but strong effects have been attributed only to one of them, the $\varepsilon 4$ allele of the $A P O E$ gene.
To investigate the involvement of other common polymorphisms in this region, the authors used an approach known as phylogenetic mapping: here, the aim is to reconstruct the most likely phylogenetic tree of a set of sequences and then to infer the mutations that best distinguish the branches of this tree with respect to time of disease onset. This approach is frequently applied in evolutionary studies, but has not been used to discover human disease mutations.

In the $23-\mathrm{kb}$ region that was analysed, only the sequence from a 10 -kb block, comprising exons 6-10 of the TOMM40 gene, yielded a reliable phylogenetic tree. TOMM40 encodes a translocase of the outer mitochondrial membrane and is only $2 \mathrm{~kb}$ from the APOE gene. The tree divides neatly into two clades, which could be distinguished by the presence of a stable poly(T) variant in a TOMM40 intron and by the enrichment of $\mathrm{AD}$ cases in one branch. In one of the clades, the longer repeat variant that was found in cis to the $\varepsilon 3$ APOE allele was correlated with earlier LOAD onset in the $\varepsilon 3$ carriers; the shorter poly $(\mathrm{T})$ variant in cis to the $\varepsilon 3 A P O E$ allele was correlated with later age of onset.

Phylogenetic mapping is suited for detecting disease-associated polymorphisms in genomic regions, such as the one containing APOE, that are diverse but evolutionarily stable, and for detecting variable repeats that would be missed unless deep sequencing is used. Although it cannot be excluded that other sites in this linkage region cause the earlier LOAD onset of $\varepsilon 3$-carrying individuals (for example, sites that were not sequenced in this study), the involvement of TOMM40 is certainly plausible, given that mitochondrial dysfunction occurs early in LOAD pathogenesis.

Tanita Casci

ORIGINAL RESEARCH PAPER

Roses, A. D. et al. A TOMM40 variable-length

polymorphism predicts the age of late-onset

Alzheimer's disease. Pharmacogenomics J.

22 Dec 2009 (doi:10.1038/tpj.2009.69)
Phylogenetic mapping is suited for detecting diseaseassociated polymorphisms in genomic regions, such as the one containing $A P O E$, that are diverse but evolutionarily stable 